\title{
Isotopía ambiental de las precipitaciones y de las aguas superficiales y subterráneas en los Andes Centrales: Revisión
}

\author{
Sonia Valdivielso(1), Enric Vázquez-Suñé(1) y Emilio Custodio ${ }^{(2)}$ \\ (1) Instituto de Diagnóstico Ambiental y Estudios del Agua (CSIC), C/ Jordi Girona 18-26, 08034, Barcelona, España. \\ sonia.valdivielso@idaea.csic.es; sonia.valdivi@gmail.com \\ (2) Real Academia de Ciencias Exactas, Físicas y Naturales de España (RAC). Dep. Ingeniería Civil y Ambiental, Univ. \\ Politécnica de Catalunya (UPC), C/ Jordi Girona 1-3, 08034 Barcelona, España.
}

\begin{abstract}
RESUMEN
Se muestra la revisión de la información existente sobre la composición isotópica del oxígeno $(\delta 180)$ y del hidrógeno $(\delta 2 \mathrm{H})$ de la molécula del agua en el sector Norte de los Andes chilenos, entre $17^{\circ} 30^{\prime}$ y $25^{\circ} 30^{\prime}$ de latitud sur. Durante el verano austral, los vientos del este favorecen la entrada de humedad atlántica, predominantemente a través de la cuenca del Amazonas, que originan más del $80 \%$ de la precipitación anual que se produce en los Andes Centrales. La cordillera de los Andes Centrales actúa como una barrera geográfica respecto a la humedad del Atlántico, siendo uno de los factores que produce en la costa norte chilena el área climática más seca de la Tierra. Se revisan brevemente los antecedentes sobre la hidrología isotópica, los que han permitido dar respuesta a cuestiones tales como el origen de las precipitaciones que recargan los acuíferos, la localización de las zonas de recarga, las posibles conexiones entre cuencas, y la identificación de los procesos que modifican la isotopía de las aguas, como la evaporación o los efectos geotérmicos. Se ha observado en las aguas superficiales, subterráneas y precipitaciones del norte de Chile, que existe un enriquecimiento en isótopos pesados en sentido norte sur, desde la región de Arica y Parinacota a la región de Antofagasta. La investigación de la isotopía de las precipitaciones y de las aguas superficiales y subterráneas es necesaria para un manejo sustentable de los recursos hídricos y para comprender el actual clima y las variaciones climáticas pasadas y futuras.
\end{abstract}

Palabras Clave: Andes, hidrología, isótopos estables, precipitación.

\section{Environmental isotope concepts of precipitation and surface water and groundwater in the Central Andes: $A$ review}

\begin{abstract}
A preliminary review of existing information on the isotopic composition of oxygen $\left(\delta^{18} \mathrm{O}\right)$ and hydrogen $\left(\delta^{2} \mathrm{H}\right)$ of the water molecule in the northern sector of the Chilean Andes, between $17^{\circ} 30^{\prime} \mathrm{S}$ and $25^{\circ} 30^{\prime} \mathrm{S}$, is presented. During the austral summer, the winds from the east favour the arrival of Atlantic humidity, dominantly through the Amazonas Basin, which produces more than the $80 \%$ of the annual precipitation. The Central Andes cordillera acts as a barrier to the wet air from the south-eastern Atlantic and in the Chilean northern coast produces the driest climatic area of the Earth. What is known about isotopic hydrology is briefly reviewed. This knowledge has allowed answers to questions such as the origin of precipitation, the possible links between basins, and the detection of processes that modify water isotopy, such as evaporation and geothermal effects. It has been observed in the surface water, groundwater and rainfall of northern Chile, that there is an enrichment in heavy isotopes in the north-south direction, from the region of Arica and Parinacota to the Antofagasta region. Continuing the research on precipitation and surface and groundwater isotopy is essential to achieve sustainable management of water resources and to understand current climate conditions and the past and future variations.
\end{abstract}

Keywords: Andes, hydrology, stable isotopes, precipitation. 


\section{Introducciónn}

El agua es fundamental para el desarrollo de la vida, para el abastecimiento humano y la realización de sus actividades económicas y para mantener los servicios ecológicos. A nivel global existe un frágil equilibrio entre los recursos de agua propia del sistema hídrico, la demanda de la actividad humana y la conservación de los ecosistemas. Su escasez de agua ha hecho necesario introducir una gestión adecuada y sostenible, lo cual requiere programas de investigación promovidos por organismos internacionales y gubernamentales, como planes estratégicos regionales, y la fundamental participación de las universidades y centros de investigación.

Los habitantes de los Andes Centrales sufren épocas de sequía en las que es difícil abastecerse y desarrollar actividades agrícolas y ganaderas, mientras que hay épocas de inundación y crecidas (huaicos) en las que el agua destruye todo a su paso. El primer paso para realizar una correcta gestión de los recursos hídricos consiste en conocer el origen de las precipitaciones que recargan los acuíferos y evaluar su magnitud.

Los isótopos estables, oxígeno $\left(\delta^{18} \mathrm{O}\right)$ e hidrógeno $\left(\delta^{2} \mathrm{H}\right)$, son una herramienta para mejorar la comprensión del funcionamiento del ciclo hidrológico a nivel global o local. Los estudios de hidrología isotópica ambiental permiten identificar el origen de las masas de aire que producen las precipitaciones (lluvia, nieve), transferencias entre cuerpos de agua comúnmente y ocasionalmente aguas hidrotermales y en relación con los magmas. También permiten identificar las áreas de recarga y su altitud y la estimación del tiempo de tránsito del agua. Así mismo, ayuda a comprender el funcionamiento hidráulico de los acuíferos, como la relación entre las aguas superficiales y subterráneas, la identificación de los efectos de la explotación intensiva y minera del agua subterránea, la intrusión marina en las zonas costeras y el origen y causas de la contaminación natural o antrópica del agua.

Friedman (1953) observó la correlación entre el contenido $\delta^{18} \mathrm{O}$ y $\delta^{2} \mathrm{H}$ en las aguas y posteriormente Craig (1961), utilizando la destilación Rayleigh, justificó una relación lineal en el diagrama $\delta^{2} \mathrm{H}$ versus $\delta^{18} \mathrm{O}$ de las aguas no evaporadas a nivel mundial y definió la Línea Meteórica Mundial (LMM) (ec. 1):

$$
\delta^{2} \mathrm{H}=8 \delta^{8} \mathrm{O}+10 \%
$$

Desde 1961, el Organismo Internacional de Energía Atómica (OIEA) en cooperación con la Organización Meteorológica Mundial (OMM), mantienen el proyecto GNIP (Global Network of Isotopes in Precipitation). Se trata de una red mundial de estaciones para el registro de datos meteorológicos básicos y la recolección de muestras de precipitaciones mensuales para su posterior análisis del contenido en isótopos estables, $\delta^{18} \mathrm{O}$ y $\delta^{2} \mathrm{H}$, y en ocasiones el isótopo radiactivo tritio $\left({ }^{3} \mathrm{H}\right)$. Dentro de este programa, en 1978, el Insti- tuto de Geocronología y Geología Isotópica (INGEIS, CONICET-UBA) estableció una Red Nacional de Colectores (RNC) de agua de precipitación, en Argentina (Dapeña and Panarello, 2005).

A partir de esta información recopilada y el modelo de destilación Rayleigh en el laboratorio, Dansgaard (1964) relacionó los parámetros geográficos (altitud, continentalidad, temperatura, latitud, cantidad) con la variación isotópica de las precipitaciones. Además, Dansgaard (1964) definió el exceso de deuterio (d) según la siguiente ecuación (ec. 2):

$$
d=\delta^{2} \mathrm{H}-8 \delta^{18} \mathrm{O}
$$

En los Andes Centrales se han realizado investigaciones sobre la circulación atmosférica actual y en el pasado en el área andina (Fuenzalida y Rutllant, 1986; Aceituno y Montencino, 1997; Garreaud, 1998; Vuille, 1999; Aravena et al., 1999; Dapeña y Panarello, 2011), el análisis de la composición isotópica del $\delta^{18} \mathrm{O}$ y $\delta^{2} \mathrm{H}$ de las precipitaciones (Chaffaut, 1992, 1998) y la influencia en la isotopía de las precipitaciones los eventos de la Oscilación del Sur-EI Niño (ENSO) (Vuille, 1999; Fiorella et al. 2015). En numerosos estudios se ha utilizado el análisis de $\delta^{18} \mathrm{O}$ y $\delta^{2} \mathrm{H}$ para identificar la altitud y las zonas de recarga y que aportan agua a los acuíferos (Houston, 2009).

En el norte de Chile (Figura 1) se han recolectado muestras de precipitaciones y de aguas superficiales y subterráneas para el análisis e interpretación de la composición isotópica $\left(\delta^{18} \mathrm{O}\right.$ y $\left.\delta^{2} \mathrm{H}\right)$ a lo largo de las últimas décadas, con diferentes objetivos. En el altiplano de la Región de Arica y Parinacota se trata principalmente los trabajos de Peña (1989), Ayala et al. (1996), Risacher et al. (1999), Herrera et al. (2006) Matraz (2015) e ICASS (2017). En la Región de Tarapacá los de Fritz et al. (1981), Aravena y Suzuki (1990), Risacher et al. (1999), Aravena et al. (1999), DIHA-PUC (2009), Scheihing (2018), Salazar et al. (1998), Uribe et al.(2015), Amphos21 (2012), Acosta et al. (2013), Achurra (2010), Achurra et al. (2011), etc. y en la II Región de Antofagasta los de Alpers y Whittemore (1990), Risacher et al. (1999), Villablanca (2009), Matraz (2014), SGA (2015), Herrera y Custodio (2013; 2014), Herrera et al. (2015; 2016; 2018), Martí (2013), entre otros. También se ha investigado en mayor detalle el origen de las aguas termales del altiplano chileno (Giggenbach, 1978; Tassi et al., 2010; Lagos, 2016; Achurra, 2010).

El objetivo de este documento es, en primer lugar, resumir la información que se ha recopilado en cuanto a la identificación del origen y la caracterización isotópica de las precipitaciones en los Andes Centrales, entre $14^{\circ} \mathrm{S}$ y $25^{\circ} \mathrm{S}$ y entre $76^{\circ} \mathrm{W}$ y $62^{\circ} \mathrm{W}$. Posteriormente, se compila la información sobre la composición isotópica de $\delta^{18} \mathrm{O}$ y $\delta^{2} \mathrm{H}$ del agua natural, tanto superficial como subterránea, con el fin de obtener una información integrada y completa de la precipitación en las tres regiones más septentrionales del altiplano chileno: Arica y Parinacota. Tarapacá y Antofagasta, entre $17,5^{\circ} \mathrm{S}$ y $25^{\circ} \mathrm{S}$ y entre $70^{\circ} \mathrm{W}$ y $67^{\circ} \mathrm{W}$. 


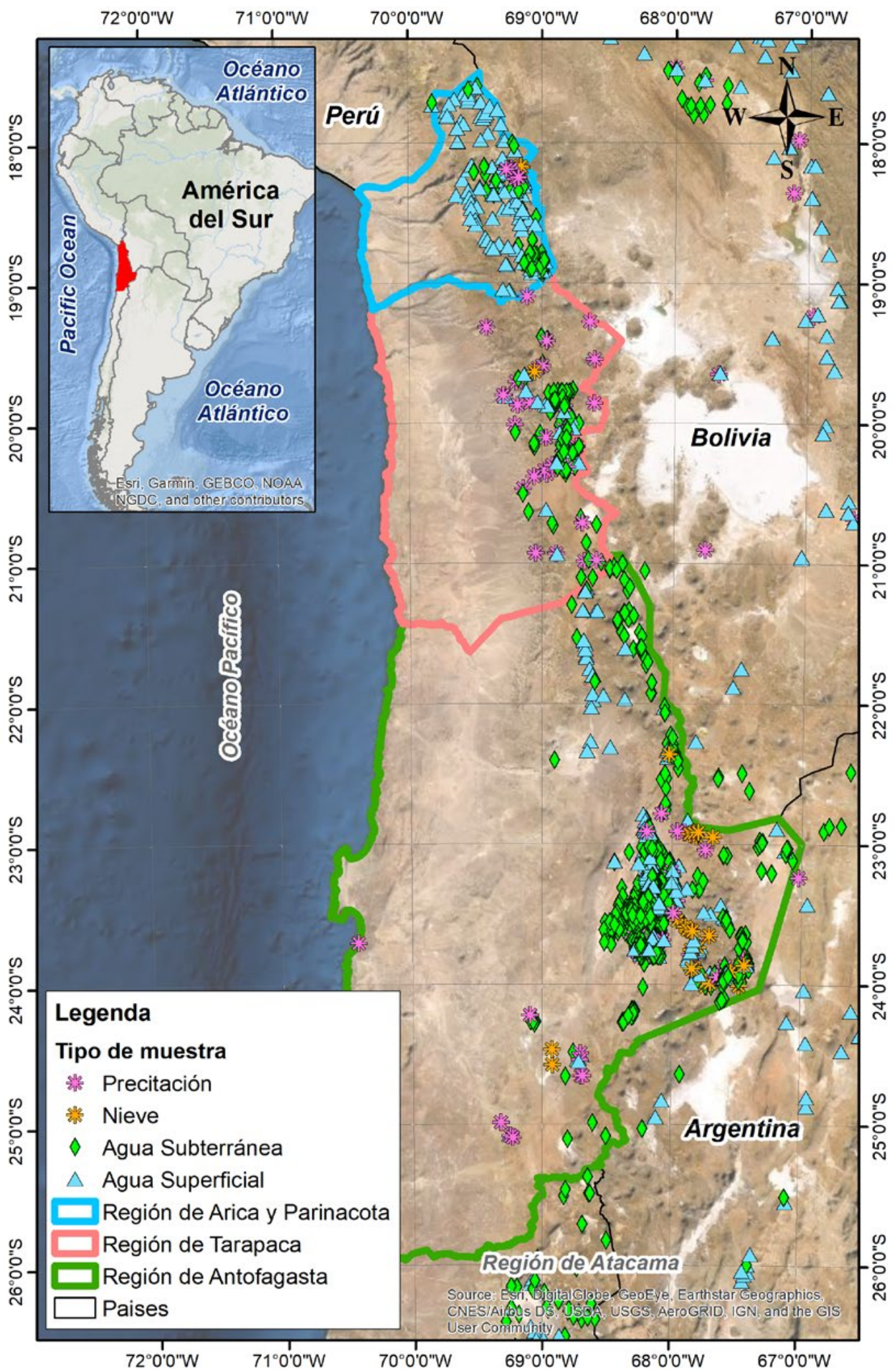

Figura 1. Mapa de ubicación de la zona de estudio y de las muestras isotópicas recopiladas.

Figure 1. Location map of the study area and the isotope samples compiled. 


\section{Metodología}

Los trabajos de recopilación y análisis hasta ahora realizados engloban los estudios, informes, tesis, congresos, artículos y otros documentos existentes, hasta donde ha sido posible, tanto la publicada como la de dominio público en instituciones como la Dirección General de Aguas de Chile (DGA), Dirección de Obras Hidráulicas de Chile (DOH), Centro de Información Recursos Hídricos de Chile (CIREN), Servicio de Evaluación Ambiental de Chile (SEA), Servicio Meteorológico Nacional de Argentina (SMN), Agencia Nacional del Agua de Perú (ANA), Servicio Nacional de Meteorología e Hidrología del Perú, Servicio Nacional de Meteorología e Hidrología de Bolivia, Organismo Internacional de Energía Atómica (OIEA/IAEA), etc. En este momento, la recopilación se ha centrado principalmente en el sector más árido de los Andes Centrales: el norte de Chile, sur de Perú, oeste de Bolivia y noroeste de Argentina. En este documento se presenta la información relacionada con el altiplano del norte chileno.

Las muestras de los estudios publicados proporcionan los valores de los isótopos estables de oxígeno e hidrógeno en notación delta ( $\delta)$, en por mil (\%o), referidos al estándar SMOW (Standard Mean Ocean Water definida por Craig, 1961), mientras que en los estudios más recientes se refieren al VSMOW (Vienna Standard Mean Ocean Water). Es importante aclarar que ambos estándares de referencia (SMOW y VSMOW) se consideran equivalentes entre sí (Gonfiantini, 1978, 1981; Lin et al., 2016).

\section{Resultados y discusión}

\section{Origen de las precipitaciones en los Andes Centrales}

Las precipitaciones en los Andes Centrales se caracterizan por presentar una marcada estacionalidad. Esta variación temporal de las precipitaciones está contralada por un régimen regional de circulación monzónica durante el verano (Zhou y Lau, 1998), produciéndose hasta un $80 \%$ de las precipitaciones anuales durante el verano austral (diciembre, enero, febrero y marzo) y escasas precipitaciones, generalmente en forma de nieve en invierno. Además, se producen fenómenos cíclicos interanuales que provocan una intensificación o debilitamiento de esta estacionalidad.

Durante el verano austral, en la troposfera superior (200 hPa), Guzman y Schwerdtfeger (1965) identificaron un anticiclón. Este sistema de alta presión se justificó gracias al calor latente liberado en las violentas tormentas eléctricas que caracterizan las condiciones climáticas del altiplano andino y a un fuerte calentamiento diabático sobre el altiplano (Zhou y Lau, 1998). A este fenómeno atmosférico se le denomina Alta Boliviana. Fuenzalida y Rutllant (1986) observaron que las precipitaciones estivales llegan precedidas de viento del este, con un alto contenido de vapor atmosférico.
Las húmedas masas de aire procedentes del este de los Andes Centrales se originan en el océano Atlántico y son arrastradas por los vientos alisios a través de la cuenca amazónica, con precipitaciones de tipo convectivo (Aravena et al. 1989, 1999). Vuille (1999) considera que la distribución temporal y espacial de las precipitaciones está influenciada por la Alta Boliviana, aunque Fiorella et al. (2015) cuestionaron la relación entre la Alta Boliviana, el origen de las masas de aire y la cantidad y la composición isotópica de las precipitaciones. Samuels-Crow et al. (2014) observaron que la convección monzónica controla los isótopos del vapor atmosférico en el verano austral, mientras que la mezcla advectiva a gran escala controla los isótopos del vapor atmosférico durante el invierno austral.

La cordillera oriental de los Andes Centrales bloquea las masas de aire que proceden de la cuenca del Amazonas, de modo que estas masas de aire se canalizan por el este de los Andes hacia el sur, a la cuenca de la Plata, en Argentina (Romero et al., 2019). Esta situación atmosférica se denomina chorro de niveles bajos sudamericano (South American Low-Level Jet, SALLJ) (Silva et al., 2010). Esta corriente en chorro que transporta el vapor reciclado desde el Amazonas es responsable de los excesos de deuterio mayores a 15 $\%$ medidos en las precipitaciones del noroeste de Argentina y su presencia fue validada con un modelo de trayectorias (Dapeña et al., 2005; González et al., 2009). En el sector del noroeste de Argentina se producen muchos fenómenos interrelacionados que se resumen en (Marengo et al., 2012).

Los años en los que se produce los eventos de la Oscilación Sur-EI Niño (ENSO), el régimen de precipitaciones varía (Vuille and Werner, 2005). Durante los veranos australes en los que se produce el evento EI Niño, las precipitaciones son menores en el altiplano al promedio de las lluvias que se producen durante los veranos sin evento (Aceituno, 1996), excepto en el desierto de Atacama donde aumentan las precipitaciones. Vuille (1999) confirmó la influencia de un evento El Niño en la zona del volcán Sajama, debido a un aumento del viento desde el oeste durante estos periodos de menor precipitación. Los veranos húmedos están asociados a La Niña. Houston (2009) determinó que el $62 \%$ de toda recarga que se produce en las cuencas Linzor (Región de Antofagasta) se concentran en los episodios de La Niña, que se producen el $23 \%$ de los años. Por lo tanto, la recarga difusa en estas cuencas altiplánicas no es un fenómeno lineal.

Durante el invierno austral (junio, julio, agosto), en la troposfera superior $(200 \mathrm{hPa})$, se determinó que el flujo de viento dominante tiene una componente de oeste a este, desde el océano Pacífico (Guzman y Schwerdtfeger, 1965; Fuenzalida y Rutllant, 1986; Vuille, 1999). Vuille (1999) identificó una reducción de la presión atmosférica y de las temperaturas y un aumento de la humedad relativa del aire durante las precipitaciones en el invierno austral. Las nevados son fenómenos bastante regulares en la cordi- 
Ilera occidental, particularmente al este del salar de Atacama (Vuille, 1999; Vuille and Baumgartner, 1998; Saavedra et al., 2017; Saavedra et al., 2018). Este resultado es coherente con el modelo numérico de mesoescala para simular y diagnosticar los procesos regionales que vinculan las condiciones locales y las de gran escala la climatología de los Andes Centrales (Garreaud, 1998). Aravena et al. (1999) observaron que las tormentas de invierno se generan por actividad frontal de aire polar con aire cálido procedente del océano Pacífico. Las precipitaciones del invierno austral no tienen relación directa con el ENSO (VuiIle and Ammann, 1997). Vuille y Keimig (2004) observan que las épocas húmedas y secas no se producen siempre en ambas cordilleras a la vez.

En el caso particular del salar de Atacama, Houston y Hartley (2003) y Houston (2006) analizaron los diferentes factores que provocan las condiciones de la hiperaridez de este sector. Los factores son: el efecto de continentalidad, que se debe a la distancia al Amazonas (origen masas de humedad); el efecto sombra de los Andes, que dificultan el paso de los vientos del este; el efecto zonal, la circulación Hadley reduce la convección (precipitación); y el efecto oceánico, corriente fría peruana produce inversión térmica atrapando la humedad por debajo de los $80 \mathrm{msnm}$.

\section{Isotopía de las aguas en el altiplano del norte de Chile}

Albero y Panarello (1980) establecieron la primera línea meteórica de Sudamérica (ec. 3), analizaron el contenido de tritio de las precipitaciones y verificaron que existe un efecto de cantidad en la composición isotópica de $\delta^{18} \mathrm{O}$ y $\delta^{2} \mathrm{H}$ de las precipitaciones.

$$
\delta^{2} H=(8,27 \pm 0,53) \delta^{18} \mathrm{O}+(11,66 \pm 2,61) \%
$$

En el norte de Chile se ha definido la línea meteórica local por Aravena et al. (1999) (ec. 4) y recientemente por Boschetti et al. (2019) (ec. 5)

$$
\begin{gathered}
\delta^{2} H=7,8 \delta^{18} \mathrm{O}+9,7 \% \\
\delta^{2} H=(7,93 \pm 0,15) \delta^{18} \mathrm{O}+(12,3 \pm 2,1) \%
\end{gathered}
$$

En la cordillera oriental de los Andes Centrales, a partir de muestras de precipitaciones anuales y mensuales en dos transectos desde la selva amazónica hasta el Altiplano boliviano y mediante el modelo numérico basado en el proceso de condensación adiabática de Rayleigh, Gonfiniantini et al. (2001) definieron el gradiente del exceso del deuterio respecto a la altitud, el cual aumenta con la altitud y es mayor que $+10 \%$ o (valor global). Fiorella et al. (2015) determinaron que el mayor factor modificador de la composición isotópica de las precipitaciones es la altitud y que las precipitaciones que se producen durante el verano afectados por el evento de El Niño son escasas y presentan enriquecimiento en isótopos pesados, mien- tras que si se produce el evento La Niña son precipitaciones empobrecidas en isótopos pesados.

Scheihing (2018) considera que la composición isotópica de $\delta^{18} \mathrm{O}$ y $\delta^{2} \mathrm{H}$ de las precipitaciones del flanco oriental de los Andes refleja los efectos de cantidad y de altitud, mientras que las del lado occidental son una mezcla de masas de aire, es decir, masas del este $y$ del oeste.

Se analizaron los antecedentes relacionados con la composición isotópica de las aguas del Altiplano chileno en las 3 regiones más septentrionales del país.

\section{Región de Arica y Parinacota}

La región de Arica y Parinacota (XV Región) es el territorio chileno más septentrional. Limita al norte con Perú, al este con Bolivia, al sur con la región de Tarapacá y al oeste con el océano Pacífico.

Peña (1989) tomó muestras de nieve en dos "penitentes" del volcán Parinacota en octubre de 1983, que muestran enriquecimiento en las que tienen mayor efecto de sublimación relativo al de fusión. En la cuenca del río Lauca y del lago Chungará, Ayala et al. (1996) analizaron el $\delta^{18} \mathrm{O}$ y $\delta^{2} \mathrm{H}$ de 30 muestras de ríos, lago, lagunas, vertientes y pozos. Risacher et al. (1999) analizaron isotópicamente el lago Chungará, la cuenca del río Lauca y la cuenca del salar de Surire. Mediante las composiciones isotópicas de $\delta^{18} \mathrm{O}$ y $\delta^{2} \mathrm{H}$ y su relación con las concentraciones de cloruro de las aguas, Herrera et al. (2006) identificaron la existencia de conexión subterránea por flujo lateral desde el lago Chungará hacia las lagunas Cotacotani. Matraz (2015) caracterizó las aguas superficiales y subterráneas de la cuenca del río Lauca. ICASS (2016) realizó el primer muestreo hidroquímico e isotópico en toda la región de Arica y Parinacota y se analizó la variación temporal y espacial de la composición isotópica. Tassi et al. (2010) identificaron interacciones de fluidos de origen profundo con agua meteórica a poca profundidad en las manifestaciones hidrotermales del salar de Surire. Lagos (2016) observó que las aguas subterráneas frías se relacionan con la meteorización de rocas silicatadas, mientras que las aguas termales están afectadas por salmueras del lago Surire.

\section{Región de Tarapacá}

La región de Tarapacá (I Región) está al sur de la región de Arica y Parinacota y al norte de la región de Antofagasta y limita al este con Bolivia y al Oeste con el océano Pacífico. Contiene La Pampa delTamarugal, que es el mayor acuífero de Chile, en un área hiperárida con aporte de las vertientes del lado andino. Está poblada, por lo que el acuífero ha sido estudiado en numerosas ocasiones.

Salazar et al. (1998) determinaron que no existe una conexión entre el salar del Huasco y el acuífero de Pica y que la cota mínima de recarga en el acuífero de Pica es de $3850 \mathrm{~m}$ snm. Uribe et al. (2015) cuan- 
tificaron la distribución espacial y temporal de la recarga en el salar del Huasco, combinando la caracterización isotópica de las aguas, el contenido en ${ }^{14} \mathrm{C}$ y la hidroquímica y el modelado de precipitación-escorrentía; descartaron la conexión entre el acuífero Salar del Huasco y los manantiales de Pica. A las mismas conclusiones llegaron Amphos21 (2012) y Acosta y Custodio (2008) y Acosta et al. (2013). Cifuentes et al. (2014) realizaron un muestreo para el análisis químico e isotópico de las aguas en el sector de Pica. Estas relaciones se han actualizado en el trabajo de Lictevout (2018).

En la Pampa del Tamarugal, a partir de las muestras recolectadas entre 1972 y 1974, Fritz et al. (1981) definieron la recta meteórica local (ec. 6) y posteriormente Salas et al. (1998) (ec.7):

$$
\begin{aligned}
& \delta^{2} H=7,8 \delta^{18} \mathrm{O}+10,3 \% 。 \\
& \delta^{2} H=7,7 \delta^{18} \mathrm{O}+9,6 \% \text { o }
\end{aligned}
$$

Magaritz et al. (1985) determinaron un exceso de deuterio del $+13 \%$ en las precipitaciones, mientras que es de $+5 \%$ en las aguas de vertientes y los ríos Tarapacá y Aroma. Salazar et al. (1998) identificaron un aligeramiento isotópico de las precipitaciones asociado al efecto cantidad en la Pampa del Tamarugal. Grilli et al. (1999) diferenciaron la composición isotópica de las precipitaciones en función de la altitud, con un contenido de $\delta^{18} \mathrm{O}$ de las aguas de lluvia mayor (más pesado) que $-11 \%$ a altitud menor a 3500 $\mathrm{m}$ snm. Acosta y Custodio (2008) constataron que el gradiente isotópico altitudinal del $\delta^{18} \mathrm{O}$ de las aguas subterráneas del área de Collacagua-Salar del Huasco-Pica-Pampa del Tamarugal es de aproximadamente $-0,2 \% / 100 \mathrm{~m}$

Cifuentes et al. (2015) realizaron un muestreo de aguas subterráneas, superficiales, vertientes y lluvias de la Pampa delTamarugal. Jayne et al. (2016), combinando un modelo bidimensional de agua subterránea y flujo de calor con observaciones de campo y valores del gradiente altitudinal del $\delta^{18} \mathrm{O}$ en aguas superficiales y subterráneas a escala regional, dedujeron que existe una recarga desde las cuencas altiplánicas hacia la Pampa del Tamarugal, pero con diferentes velocidades de flujo. Risacher et al. (1999) y Amphos21 (2012) muestrearon isotópicamente los salares de Coposa, del Huasco y de Michincha, para estudiar las posibles transferencias de aguas internas y hacia los salares de Bolivia, la Quebrada Chacarillas y Pampa del Tamarugal. Scheihing (2018) ha recopilado 514 muestras de precipitaciones y de aguas superficiales y subterráneas distribuidas por la región con el fin de caracterizarlas isotópicamente e identifican las zonas de recarga.

Las aguas termales de Puchuldiza-Tuja y de Pampa Lirima fueron muestreadas y analizadas por Tassi et al. (2010) y las de Pampa Lirima por Achurra (2010) y Achurra et al. (2011) para establecer hidroquímica e isotópicamente el área de recarga.

\section{Región de Antofagasta}

La región de Antofagasta (II Región) se encuentra al sur de la Región de Taracapá y al norte de la región Atacama y limita al este con Argentina y al oeste con el océano Pacífico. En esta región existen numerosos estudios debido a la gran actividad minera.

Se ha estudiado isotópicamente la Cordillera de la Costa y se ha determinado que las muy escasas Iluvias se originan en el océano Pacífico y no rebasan la Cordillera hacia el este, con agua subterránea que responde a paleorecarga (Herrera y Custodio, 2013, 2014; Herrera et al., 2018). En la cuenca del río Loa Alto, desde su nacimiento hasta Calama, Aravena y Suzuki (1990), Matraz (2014) y Martí (2013) determinaron que el río Loa está controlado por el agua subterránea de varios orígenes y por afluentes que muestran cierta diferenciación de isótopos debido a la evaporación (río Salado, embalse Conchi, etc.). En las cuencas altiplánicas que se encuentran al este del salar de Atacama se han recogido muestras de precipitación, nieve y aguas superficiales y subterráneas para analizar el contenido de $\delta^{18} \mathrm{O}$ y $\delta^{2} \mathrm{H}$. Se han investigado posibles conexiones entre cuencas (Lorca, 2011; Herrera et al., 2015, 2016), zonas de recarga (Alpers y Whittemore. 1990; Risacher et al., 1999; Villablanca, 2009; DIHA-PUC, 2009) y el posible impacto de la extracción de aguas subterráneas (WMCL., 2007). Las aguas superficiales y subterráneas de la cuenca del salar de Atacama fueron recolectadas y analizadas por SGA (2015). Amphos21 (2018) ha reinterpretado la información recopilada en diversos estudios. Rissmann et al. (2015) investigaron el sector Monturaqui-Negrillar-Tilopozo. Hay numerosos datos aún no publicados. El campo geotérmico EI Tatio fue investigado por Giggenbach (1978) a partir de muestras de agua de manantiales, pozos y lluvia y también por Cortecci et al. (2005). Tassi et al. (2010) analizaron el hidrotermalismo de Pampa Apacheta, EI Tatio y Torta de Tocorpuri.

En el altiplano de la región de Antofagasta, Chaffaut (1998) definió la recta meteórica local (ec. 8) y posteriormente Villablanca (2009) (ec.9):

$$
\begin{array}{cc}
\delta^{2} H=8,15 \delta^{18} \mathrm{O}+15,3 \% & \text { (ec. } 8) \\
\delta^{2} H=7,95 \delta^{18} \mathrm{O}+15,89 \% 。 & \text { (ec.9) }
\end{array}
$$

Risacher et al. (1999), a partir de los datos isotópicos del salar de Surire y las precipitaciones analizadas por Mardones (1997) en el salar de Ascotán (Región II Atacama), determinaron la temperatura de estas precipitaciones según los valores isotópicos (ec. 10).

$$
\left.\delta^{18} \mathrm{O}=0,521 T-14,96 \% \text { (ec. } 10\right)
$$

donde $T$ es la temperatura en grados centígrados $\left({ }^{\circ} \mathrm{C}\right)$.

La interpretación de los datos en áreas montañosas a tener en cuenta el efecto de ladera, es decir la acumulación de recarga según el flujo. Para su estudio se ha realizado un estudio analítico, en parte inspirado en los procesos en estas áreas (Custodio y Jódar, 2016). 


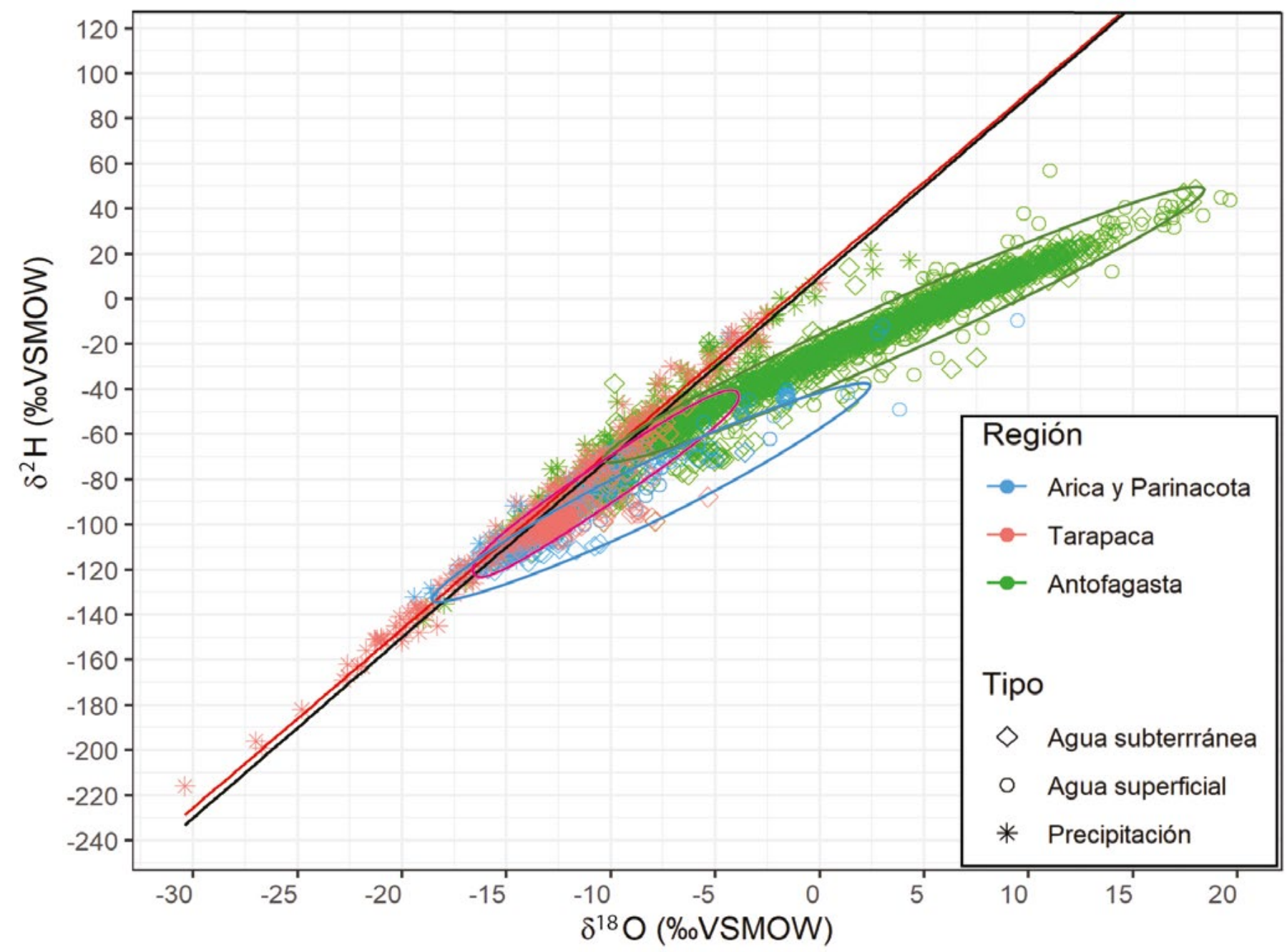

Figura 2. $\delta^{18} \mathrm{O}$ y $\delta^{2} \mathrm{H}$ de las muestras de agua y precipitaciones recopiladas en el norte de Chile. La línea negra corresponde a la LMM y la línea roja a la LML definida por Boschetti et al. (2019) (ec. 5).

Figure 2. $\delta^{18} \mathrm{O}$ and $\delta^{2} \mathrm{H}$ in the water and precipitation samples in the north of Chile. The black line corresponds to the LMM and the red line to the LML defined by Boschetti et al. (2019).

\section{Conclusiones y futuros trabajos}

Se dispone de numerosos estudios sobre la precipitación, en algunos casos de nieve, y de las aguas subterráneas en los Andes Centrales, que en buena parte están aquí referenciados. Aunque la distribución espacial y temporal de los datos es muy irregular y no hay estaciones de las redes internacionales ni nacionales, es posible obtener una información a gran escala de los principales procesos asociados a la precipitación y a la recarga. Gran parte de la escasa y a veces casi nula precipitación es de origen atlántico y la de origen pacífico se limita a las raras lluvias de la franja costera. La recarga a los acuíferos es en parte paleorecarga en las áreas más áridas, pero es renovable en el Altiplano y laderas, con recarga que es una fracción notable de la precipitación allí donde hay nieve temporal y se tienen suelos volcánicos recientes.
Los datos isotópicos de $\delta^{18} \mathrm{O}$ y $\delta^{2} \mathrm{H}$ de las precipitaciones y aguas superficiales y subterráneas recopilada en los antecedentes publicados anteriormente, muestran una variación espacial en el norte del altiplano chileno. La composición isotópica de $\delta^{18} \mathrm{O}$ y $\delta^{2} \mathrm{H}$ de las aguas muestran un progresivo enriquecimiento en isótopos pesados desde la región de Arica y Parinacota hacia el sur, región de Antofagasta (Figura 2). Los valores $\delta^{18} \mathrm{O}$ y $\delta^{2} \mathrm{H}$ van desde $-17 \%$ y $-130 \%$ respectivamente en las aguas tomadas en la región de Arica y Parinacota hasta -3 y $-40 \%$ respectivamente en la región de Antofagasta. Esta variación en la composición isotópica de las aguas y precipitaciones es el objeto de futuros estudios, ya que estará relacionado con parámetros geográficos (altitud, continentalidad, temperatura, latitud), el efecto de cantidad de las precipitaciones o/y origen y trayectorias de las masas de aire que producen las precipitaciones en el altiplano del norte de Chile.

Se proporciona una información integrada sobre las 
fuentes de datos sobre la composición isotópica de las precipitaciones y aguas en los Andes Centrales. Los resultados son esenciales pare entender evaluar y formar modelos conceptuales de la recarga a los acuíferos. Por esa razón, los esfuerzos que se realicen para mejorar la situación actual y mantener redes de monitoreo redundará sin duda en una mejor gestión de los escasos recursos de agua, la conservación de los notables valores ambientales ligados al agua subterránea y al desarrollo humano, incluyendo a la minería sostenible. La colaboración entre los distintos organismos y entidades nacionales y supranacionales es importante y su consecución puede ser un beneficio adicional.

\section{Referencias}

Aceituno, P., 1996. Elementos del clima en el Altiplano Sudamericano. Revista Geofísica, 44, 37-55.

Achurra, L. 2010. Estudio hidrogeoquímico sobre la interacción de las aguas subterráneas profundas y someras en Pampa Lirima, Norte de Chile. Tesis Maestría. FCIHS, Universitat Politècnica de Catalunya. Barcelona.

Achurra, L., Custodio, E., Aguirre, I., Arcos, R. and Clavero, J. 2011. Estudio hidrogeoquímico en la cuenca altiplánica de Pampa Lirima, Andes Centrales (Chile). VII Congreso Argentino de Hidrogeología y $\checkmark$ Seminario Hispano-Latinoamericano. Salta, Argentina: 276-283.

Acosta, O. and Custodio, E., 2008. Impactos ambientales de las extracciones de agua subterránea en el salar de Huasco (norte de Chile). Boletín Geológico y Minero, 119 (1): 33-50.

Acosta, O., Guimerà, J., Custodio, E., Ansón, I. and Delgado, J.L. 2013. Contribución al conocimiento de la hidrogeología de las cuencas intraandinas del $N$ de Chile. En Agua Subterránea Recurso Estratégico. Universidad de La Plata (Edulp). 1: 118-125.

Albero, M.C. and Panarello, H.O., 1980. Tritio e isótopos estables en aguas de precipitaciones en América del Sur. Interamerican Symposium on Isotope Hydrology. Bogotá, Colombia.

Alpers, C. and Whittemore, D., 1990. Hydrogeochemistry and stable isotopes of groundwater and surface waters from two adjacent closed basins, Atacama Desert, northern Chile. Applied Geochemistry, 5: 719-734.

Amphos21. 2012. Estudio de los tiempos de residencia del agua subterránea en el entorno de la Compañía Minera Doña Inés de Collahuasi. Chile.

Amphos21. 2018. Estudio de modelos hidrogeológicos conceptuales integrados, para los salares de Atacama, Maricunga y Pedernales Modelo Hidrogeológico Consolidado Cuenca Salar de Atacama. Comité de Minería No Metálica CORFO. Chile: 1-368.

Aravena, R., 1995. Isotope hydrology and geochemistry of northern Chile ground waters. Bulletin de I'Institut Français d'Etudes Andines 24(3):495-503.
Aravena, R., Peña, H., Grilli, A., Suzuki, O. and Mordeckai, M., 1989. Evolución isotópica de las lluvias y origen de las masas de aire en el Altiplano chileno. Estudios de Hidrología Isotópica en América Latina. IAEA-TECDOC-502. IAEA, Vienna: 129-142.

Aravena, R., A. and, Suzuki, O. 1990. Isotopic evolution of river water in the Northern Chile Region. Water Resources Research, 26(12): 2887-2895.

Aravena, R., Suzuki, O., Peña, H., Pollastri, A., Fuenzalida, H., and Grilli, A., 1999. Isotopic composition and origin of the precipitation in Northern Chile. Applied Geochemistry, 14: 411- 422.

Ayala, Cabrera y Asociados Ltda. 1996. Estudio de aguas subterráneas sector río Lauca Arica, I Región, Etapa 1. Empresa de Servicios Sanitarios de Tarapacá S.A.. Chile: 1-106.

Boschetti, T., Cifuentes, J., lacumin, P., and Selmo, E., 2019. Local meteoric water line of northern Chile $\left(18^{\circ} \mathrm{S}-30^{\circ} \mathrm{S}\right)$ : An application of error-in-variables regression to the oxygen and hydrogen stable isotope ratio of precipitation. Water (Switzerland) 11: 791.

Chaffaut, I. 1992. Precipitations d'altitude, eaux souterraines et changements climatiques de l'altiplano Nord-chilien. Thesis, Université de Paris Sud, U.F.R Scientifique d'Orsay: 1-597.

Chaffaut, I. Coudrain-Ribstein, A., Michelot, J.L., and Pouyaud, B. 1998. Précipitation d'altitude du nordChili, isotopiques origine des sources de vapeur et données. Bulletin de I'Institut Français d'Etudes Andines . 1998, 27, 367-384

Cifuentes, J., López, L. and Troncoso, R., 2014. Caracterización de hidrogeológica de la parte baja-central de la Pampa del Tamarugal y Pica, Región de Tarapacá, Chile. Servicio Nacional de Geología de Chile. pp. 7.

Cifuentes, J., Cervetto, M., López, L., Fuentes, F., Feuker, P. and Espinoza, C. 2015. Análisis preliminar de isótopos estables en aguas subterráneas, superficiales y lluvia de la Pampa del Tamarugal. Depart. Geología Aplicada, Servicio Nacional de Geología y Minería. pp 5. Chile.

Cortecci, G., Boschetti, T., Mussi, M., Herrera, C., Mucchino, C. andBarbieri, M., 2005. New chemical and original isotopic data on waters from El Tatio geothermal field in northern Chile. Geochemical Journal, 39(6): 547-571.

Craig, H., 1961. Isotope variations in meteoric waters. Science, 133: 1702-1703.

Custodio, E. and Jódar, J. 2016. Simple solutions for steady-state diffuse recharge evaluation in sloping homogeneous unconfined aquifers by means of atmospheric tracers. Journal of Hydrology, 540: 287-305.

Dansgaard, E., 1964. Stable isotopes in precipitation. Tellus 16: 436-468.

Dapeña, C. and Panarello, H.O., 2005.Evolución y estado actual de la Red Nacional de Colectores de Isótopos en Precipitación de la República Argentina. XVI Congreso Geológico Argentino, La Plata, Actas II: 635-642. 
Dapeña, C. and Panarello, H.O. 2011. Composición isotópica de las precipitaciones en el noroeste argentino. VII Congreso Argentino de Hidrogeología y V Seminario Hispano-Latinoamericano Sobre Temas Actuales de la Hidrología Subterránea. Argentina. 385- 392.

DIHA-PUC, 2009. Levantamiento hidrogeológico para el desarrollo de nuevas fuentes de agua en áreas prioritarias de la zona norte de Chile, regiones $X V$, I, II y III VIII. Pontificia Universidad Católica de Chile y Dirección General de Aguas, Santiago, Chile.

Fiorella, R. P., Poulsen, C.J., Pillco, R. S., Barnes J.B., Tabor, C.R. and Ehlers T.A. 2015. Spatiotemporal variability of modern precipitation ${ }^{18} \mathrm{O}$ in the central Andes and implications for paleoclimate and paleoaltimetry estimates. Journal of Geophysical Research: Atmospheres, 120, 4630-4656.

Friedman, I., 1953. Deuterium content of natural water and other substances. Geochimica et Cosmochimica Acta 4:89-103.

Fritz P, Suzuki O., Silva C. and Salati E. 1981. Isotope hydrology of groundwaters in the Pampa del Tamarugal, Chile. Journal of Hydrolology, 58(12):161-184.

Fuenzalida, H., and J. Rutllant, 1986: Estudio sobre el origen del vapor de agua que precipita en el invierno altiplánico. Universidad de Chile y Ministerio de Obras Públicas de Chile. Pp 53.

Garreaud, R. D., 1998. Multiscale analysis of the summertime precipitation over the Central Andes. Monthly Weather Review 127: 901-920.

Giggenbach, W., 1978. The isotopic composition of waters from the ElTatio geothermal field, Northern Chile. Geochimica et Cosmochimica Acta, 42: 979988.

Gonfiantini, R., 1978. Standards for stable isotope measurements in natural compounds. Nature 271, 534-536.

Gonfiantini, R., Roche, M-A., Olivry, J-C-, Fontes, J.C., and Zuppi, G.M. 2001.The altitude effect on the isotopic composition of tropical rains. Chemical Geology, 181(1-4): 147-167

Grilli, A.; Aguirre, E.; Durán, M.; Townsend, F. and González, A. 1999. Origen de las aguas subterráneas del sector Pica - Salar del Huasco, Provincia de lquique, I región de Tarapacá. XIII Congreso de Ingeniería Sanitaria y Ambiental AIDIS-Chile, Antofagasta. pp 18.

González, M., Dapeña, C., Cerne, B., Sanchez-CcoylIo, O., Freitas, S. and Silva Dias, P.L. and Panarello, H., 2009. Verification of the geographical origin of modeled air-mass trajectories by means of the isotope composition of rainwater during the SALLJEX experiment. Environmental Fluid Mechanisms. 9 (4): 389-407.

Guzman, G.L. and Schwerdtfeger, W., 1965. The role of latent and sensible heat for the development of a high pressure system over the subtropical Andes in the summer. Meteorologische Rundschau, 18: 69-75.
Herrera, C., Pueyo, J., Sáez, A. and Valero-Garcés, B., 2006. Relación de aguas superficiales y subterráneas en el área del lago Chungará y lagunas de Cotacotani, norte de Chile: un estudio isotópico. Revista de Geología 33 (2): 299-325.

Herrera, C. and Custodio, E. 2013. Origen de los manantiales costeros del entorno árido de Antofagasta, Norte de Chile. X Simposio de Hidrogeología. Granada. Hidrogeología y Recursos Hidráulicos. AlH-GE. Madrid, XXX: 943-952.

Herrera, C. and Custodio, E. 2014. Origin of waters from small springs located at the northern coast of Chile, in the vicinity of Antofagasta. Andean Geology 41(2): 314-341.

Herrera, C., Custodio, E., Urrutia, J., Urqueta, H., Sarmiento, A. and Gamboa, C. 2015. Caracterización de las aguas subterráneas de una pequeña cuenca endorreica en el Altiplano volcánico del Norte de Chile. En: M.C. Cabrera et al. (eds.), Estudio, Aprovechamiento y Gestión del Agua enTerrenos e Islas Volcánicas". Las Palmas de GC: IGME/AIH- GE: I 191-198.

Herrera, C., Custodio, E., Chong, G., Lambán, L.J., Riquelme, R., Wilke, H., Jódar, J., Urrutia, J., Urqueta, H., Sarmiento, A., Gamboa, C. and Lictevout, E. 2016 Groundwater flow in a closed basinwith a saline shallow lake in a volcanic area: LagunaTuyajto, northern Chilean Altiplano of the Andes. Science of the Total Evironment, 541: 303-318.

Herrera, C., Gamboa, C., Emilio Custodio, E., Jordan, T., Godfrey, L., Jódar, J., Luque, J.A., Jimmy Vargas, J., and Sáez, A. 2018 Groundwater origin and recharge in the hyperarid Cordillera de la Costa, Atacama Desert, northern Chile. Science of the Total Evironment 624.: 114-132.

Houston, J and Hartley, A. 2003.The central Andean west-slope rainshadow and its potential contribution to the origin of hyper-aridity in the Atacama desert. International Journal of Climatology. 23: 1453-1464.

Houston, J. 2006. Variability of precipitation in the Atacama desert: its causes and hydrological impact, International Journal of Climatology. 26: 2181-2198.

Houston. J, 2009. A recharge model for high altitude, arid, Andean aquifers. Hydrological Processes. 23, 2383-2393.

ICASS., 2016. Análisis integral de soluciones a la escasez hídrica, región de Arica y Parinacota. Dirección General de Aguas, S.I.T. No 410. Chile. pp 1396.

Jayne. R., Pollyea, R., Dodd, J., Olson, E. and Swanson, S. 2016. Spatial and temporal constraints on regional-scale groundwater flow in the Pampa del Tamarugal Basin, Atacama Desert, Chile. Hydrogeology Journal,24: 1921-1937.

Lagos, L.V. 2016. Hidrogeoquímica de fuentes termales en ambientes salinos relacionados con salares en los Andes del norte de Chile. Memoria título de geología, Univ. de Chile. pp 298.

Lictevout, E. 2018. Accès a l'eau des populations vulnerables en zone aride: un probleme de ressource, 
de gestion ou d'information? These, Université de Montpellier: 1-205.

Lin, J., Liu, Y., Yang, Y., and Hu, Z., 2016. Calibration and correction of LA-ICP-MS and LA-MC-ICP-MS analyses for element contents and isotopic ratios. Solid Earth Science. 1, 5-27.

Lorca, M.E.P. 2011. Hidrogeología e hidrogeoquímica de la cuenca de la quebrada Paipote, región de Atacama. Memoria para optar al Título de Geología, Universidad de Chile. pp 298. Chile.

Magaritz, M., Suzuki, O., Aravena, R., Peña, H., Grilli, A. and Orphanópoulos, D. 1985. Isotopic and chemical study of the water resources in the lquique Province. IAEA, Vienna.

Mardones, L. 1997. Características isotópicas de las aguas subterráneas entre los paralelos $21^{\circ}$ y $24^{\circ}$ latitud sur, con énfasis en el salar de Ascotán. Coloquio Internacional: El Recurso Agua en los Andes, Orstom - Universidad Católica del Norte, 10-13 de junio de 1997, Antofagasta.

Marengo, J.A., Liebmann, B., Grimm, A.M., Misra, V., Silva Dias, P.L., Cavalcanti, I.F.A., Carvalho, L.M.V., Berbery, E.H., Ambrizzi, T., Vera, C.S., Saulo, A.C., Nogues-Paegle, J., Zipser, E., Seth, A. and Alves, L.M., 2012. Recent developments on the South American monsoon system. International Journal of Climatology. 32, 1-21.

Martí, N. 2013. Caracteritzación hidrogeoquímica e isotópica de la cuenca del Alto Loa, Región de Antofagasta, Chile. Tesis Maestría. Dep ITCM, Universitat. Politècnica de Cartalunya. Barcelona

Matraz, 2014. Caracterización hidrogeoquímica de la cuenca del Loa Alto, Región de Antofagasta, Dirección General de Aguas, S.I.T. N³35. Chile.

Matraz, 2015.Diagnóstico de disponibilidad hídrica en la cuenca del río Lauca, región de Arica y Parinacota. Dirección General de Aguas, S.I.T. N³65. Chile. pp 460

Peña, H. 1989. Mediciones de ${ }^{18} \mathrm{O}$ y ${ }^{2} \mathrm{H}$ en "penitentes" de nieve. Estudios de hidrología isotópica en América Latina, IAEA-TECDOC 502, Vienna: 143154.

Risacher, F., Alonso, H. and Salazar, C., 1999. Geoquímica de aguas en cuencas cerradas, I, II, III Regiones, Chile. Dirección General de Aguas. S.I.T. N ${ }^{\circ}$ 51. Chile: 1-781.

Rissmann, C., Leybourne, M., Benn, C and Christenson, B. 2015. The origin of solutes within the groundwaters of a high Andean aquifer. Chemical Geology, 396: 164-181.

Salazar, C., Rojas, L. and Pollastri, A., 1998 Evaluación de recursos hídricos en el sector de Pica. Hoya de la Pampa del Tamarugal, I región. Dirección General de Aguas. S.I.T. N ${ }^{\circ}$. 48, pp 98.

Saavedra, F.A., Kampf, S.K., Fassnacht, S.R. and Sibold, J.S., 2017. A snow climatology of the Andes Mountains from MODIS snow cover data. International Journal of Climatology. 37, 1526-1539. https://doi.org/10.1002/joc.4795

Saavedra, F.A., Kampt, S.K., Fassnacht, S.R. and Sibold, J.S., 2018. Changes in Andes Mountains snow cover from MODIS data 2000-2016. The Cryosphere Discussions. 12, 1027-1046. https://doi.org/10.5194/ tc-2017-72.

Samuels-Crow, K.E., Galewsky, J., Hardy, R.D., Sharp, D.Z. and Worden, J., Braun, C., 2014. Upwind convective influences on the isotopic composition of atmospheric water vapor over the tropical Andes. Journal of Geophysical Research: Atmospheres. 119: 7051-7063.

SGA., 2015. Evaluación Impacto Ambiental. Proyecto modificaciones y mejoramiento del sistema de pozas de evaporación solar en el Salar de Atacama. Solución en Gestión Ambiental. Rockwood Lithium. Chile.

Scheihing, W., 2018. Water resources management in the Atacama Desert: pivotal insights into arid Andean groundwater systems. Tesis doctoral. Technische Universität Berlin: 1--170.

Tassi, F., Aguilera, F., Darrah, T., Vaselli, O., Capaccioni, B., Poreda R.J. and Delgado Huertas, A., 2010. Fluid geochemistry of hydrothermal systems in the Arica-Parinacota, Tarapacá and Antofagasta regions (northern Chile). J. Volcanology and Geothermal Research, 192: 1-15

Uribe, J., Muñoz, J.F. and Gironás, J., 2015. Assessing groundwater recharge in an Andean closed basin using isotopic characterization and a rainfall-runoff model: Salar del Huasco basin, Chile. Hydrogeology Journal, 23: 1535-1551.

Villablanca, D., 2009. Estudio de la relación isotópica $\delta 180 / \delta 2 \mathrm{H}$ de los manantiales en el sector de las nacientes del Loa, Región de Antofagasta. XII Congreso Geológico Chileno. 22-26.

Vuille, M., 1999. Atmospheric circulation over the Bolivian Altiplano during DRY and WET periods and HIGH and LOW index phases of the Southern Oscillation. International Journal of Climatology. 19: 1579-1600.

Vuille, M. and Ammann, C., 1997. Regional Snowfall Patterns in the High, Arid Andes. Climatic Change. High Elev. Sites 36: 181-191.

Vuille, M. and Keimig, F., 2004. Interannual variability of summertime convective cloudiness and precipitation in the central Andes derived from ISCCP-B3 data. Journal of Climate. 17: 3334-3348.

Vuille, M. and Werner, M., 2005. Stable isotopes in precipitation recording South American summer monsoon and ENSO variability: Observations and model results. Climate Dynamics. 25: 401-413.

WMCL. 2007. Evaluación de Impacto Ambiental, Pampa Colorada, Minera Escondida Ltda. Water Management Consultants Ltda.: 1-75..

Zhou, J. and Lau, K. M. 1998. Does a monsoon climate exist over South America? Journal of Climate, 11: 1020-1040.

Recibido: julio 2019

Revisado: noviembre 2019

Aceptado: enero 2020 